\title{
54 \\ Diffuse Malignant Pleural Mesothelioma: The Role of Pleurectomy
}

\author{
Jasleen Kukreja and David M. Jablons
}

Diffuse malignant pleural mesothelioma (MPM) is an aggressive tumor with dismal prognosis that has largely been associated with exposure to asbestos. As a disease of industrialized nations predominantly, it is expected to have its peak incidence around year 2020. In the United States alone, 2500 to 3000 new cases of MPM are diagnosed annually and its incidence is increasing. ${ }^{1-4}$ Based on a 20- to 50-year latency period between exposure and disease manifestation, there might still be another surge in incidence in the mid 21st century associated with asbestos exposure from the unfortunate events of September 11, 2001, at the World Trade Center in New York City. Despite important advances in our understanding of this disease, long-term survivors are rare due to delay in diagnosis and rapid disease progression. Malignant pleural mesothelioma poses a significant healthcare problem not only for patients and their caregivers, but also for industry and government in terms of the enormous cost of compensation.

Median survival for untreated patients is reported to be between 4 to 18 months from the time of diagnosis. Unlike other thoracic malignancies, patients usually succumb to locoregional disease with invasion of vital structures such as lungs, heart, great vessels, and esophagus, rather than metastases. Hence, early diagnosis is critical in the treatment of MPM. ${ }^{5,6}$ With the advent of aggressive local control such as extrapleural penumonectomy and radical pleurectomy/decortication with systemic multimodality therapies, patients are living longer but presenting with more systemic failures and metastases.
Brain metastases, for example, while once exceedingly rare in MPM, are now seen commonly.

Computed tomography (CT) is the primary modality of choice for evaluating MPM. Computed tomography findings can be used to preclude surgery in patients with obviously unresectable tumors (e.g., diffuse extension of tumor into the chest wall, mediastinum, vital organs, peritoneum, or distant metastasis). ${ }^{7}$ Magnetic resonance imaging (MRI) has been recommended occasionally as an adjunct to more accurately define invasion of the diaphragm and endothoracic fascia. ${ }^{7-9}$ Recently, flourodeoxyglucose-positron emission tomography (PET-FDG) has emerged as a new imaging modality in the evaluation of MPM with reportedly a better sensitivity, specificity, and accuracy $(97 \%, 80 \%$, and $94 \%$, respectively) for detecting all histological types of MPM lesions when compared with CT $(83 \%, 80 \%$, and $82 \%$, respectively). ${ }^{10}$ Positron emission tomography may also help ascertain the biological aggressiveness of the tumor and hence determine the prognosis in patients with MPM. In a recent study, those with higher FDG uptake had a significantly shorter survival time. ${ }^{11}$ Based on these criteria, a decision can be made whether to pursue an aggressive approach or not. More recently, fusion PET-CT scanning has replaced PET scans for a better correlation of anatomical detail with metabolic activity. Currently, however, FDG-PET based scanning is not widely used for MPM staging in the United States.

Despite the imaging modalities mentioned above, some patients still require surgical staging/ confirmation of N2 status. Video-assisted thorascopic surgery (VATS) has become the current 
gold standard for establishing a histological diagnosis, ${ }^{12}$ as well as for staging patients. In addition to stage, age, performance status, and epithelial histology have a significant impact on survival in MPM. ${ }^{13}$ The presence of chest pain, weight loss, high platelet counts $(>400 \mathrm{~K})$, low hemoglobin, high white-blood-cell count, and high lactate dehydrogenase ( $>500$ IU/L) impact negatively on patient survival. ${ }^{14}$ No clear consensus exists as to a specific biomarker or set of biomarkers that are either prognostic or can clearly direct therapy. Yet progress in understanding the molecular pathogenesis of MPM does hold promise for being able to identify in the future a specific set of genes and/or biomarkers that can predict biological aggressiveness, the chance of metastasis, and the likelihood that aggressive combined modality interventions will confer a long-term survival advantage. The science, however, is not here yet.

\subsection{Therapy}

There is no universally accepted standard therapy for MPM. Treatment options include chemotherapy, radiation therapy, surgery, or some combination of these modalities. Single-modality therapy has had limited success in the treatment of this disease. The median survival for patients receiving supportive care alone has been reported to be as low as 6 months. ${ }^{15,16}$ As in most orphan and understudied diseases, however, the interpretation of any data is fraught with significant bias and hampered by underpowered, nonrandomized, nonprospective studies. In addition, because of the nonstandardized staging system for MPM, many observations cannot be extrapolated across institutions or countries. Given that most data (with a few exceptions noted below) are from retrospective, small single-institution trials that spanned years if not decades to accrue patients with evolving staging techniques and modalities, it is difficult to draw firm conclusions regarding best treatment option(s).

\subsubsection{Single-Modality Therapy}

\subsubsection{Chemotherapy}

Up until recently, the median survival time of patients treated with chemotherapy alone, either as single agent or some combination, was no better than 7 months. ${ }^{14}$ However, newer agents, in particular gemcitabine and pemetrexed in combination with platinum analogs, have recently been shown to improve response rates and quality of life. In a phase II study, Byrne and colleagues achieved a partial response rate of $47 \%$ in patients with advanced-stage pleural mesothelioma treated with combination of cisplatin and gemcitabine. $^{17}$

Pemetrexed, a novel multitargeted anti-folate that inhibits DNA synthesis, in combination with platinum analogs in two phase I studies, achieved a partial response (PR) rates of $32 \%$ and $45 \%$, respectively. ${ }^{18-20}$ Based on the encouraging results of the aforementioned trials, Vogelzang and coworkers ${ }^{21}$ conducted a phase III trial to determine whether combination pemetrexed/cisplatin conferred a survival advantage in patients with MPM compared with cisplatin alone.

In this multicenter, international, randomized study, 456 patients with unresectable MPM were randomized to receive either a combination of pemetrexed and cisplatin or cisplatin alone. Combination pemetrexed/cisplatin significantly improved time to relapse (5.7 months vs. 3.9 months; $p=0.001$ ) and, most importantly, survival (12.1 months vs. 9.3 months; $p=0.02)$. The 2.8-month survival benefit in the combination chemotherapy group translated into an improved hazard ratio of 0.77 or, conversely, a meaningful relative risk of death reduction of $23 \%$. Therefore, combination pemetrexed/cisplatin is now routinely used for advanced-stage biopsy proven MPM (level of evidence 1a).

\subsubsection{Radiation}

The role of radiation as single-modality therapy is difficult to evaluate due to the fact that radiation has generally been used as part of either bimodality or multimodality therapy in conjunction with chemotherapy, surgery, or both. Effectiveness of radiation therapy alone is limited by the comparatively radio-resistant nature of the disease, the high doses, and/or the extensive target areas required. Radiation, however, has been used to palliate areas of symptomatic tumor invasion. ${ }^{22}$ Radiotherapy has also been used effectively to prevent biopsy tract site malignant 
seeding. In a recent randomized study comparing those receiving daily sessions of local radiotherapy to the invasive/diagnostic entry site with those receiving no radiation, none of the patients treated with radiation developed entry tract metastasis as opposed to $40 \%$ of the untreated that did. ${ }^{23}$ This study supports the use of early local radiotherapy in preventing malignant seeding at the biopsy site or chest-tube site (level of evidence $1 b$ ).

\subsubsection{Surgery}

Similar to chemotherapy and radiation, surgery as a single-modality therapy has not been overly successful in the treatment of MPM. Furthermore, only $20 \%$ to $30 \%$ of patients at the time of presentation are amenable to resection. Of those, $20 \%$ are unresectable at the time of operation. Because MPM is predominantly a loco-regional disease, surgical strategies have focused on loco-regional control. Current surgical therapy for patients with MPM includes radical surgery (extrapleural penumonectomy), debulking surgery (pleurectomy/decortication), and palliation (drainage effusion and pleurodesis). Surgery in MPM, like any operation for cancer, has the most benefit when an $\mathrm{R} 0$ or nearly $\mathrm{R} 0$ resection can be achieved. R0 status after resection has been shown by a few groups to confer a survival advantage compared to those that received lesser degree of resection. This philosophy has significant import in approaching patients with MPM for surgical treatment. While palliative decortications alone can make patients less dyspneic and offer better quality of life in the short term, no real evidence exists to suggest that it confers any survival advantage. Radical pleurectomy (visceral and parietal) with skeltonization of hilar and mediastinal pericardial and diaphragmatic pleura, however, can lead to a meaningful cytoreduction and chance of increased survival if R0 status can be achieved. The evidence of data, however, is limited.

Historically, extrapleural pneumonectomy (EPP) entailing en bloc resection of the pleura, lung, ipsilateral hemidiaphragm, and pericardium has been limited by high operative mortality (30\%). ${ }^{24}$ As a result, pleurectomy/decortication (P/D) that only removes all gross pleural disease without removing the underlying lung, pericardium, or diaphragm has been preferred over EPP by some surgeons due to lower associated morbidity and mortality (1.5\% to $5 \%) .{ }^{25}$ However, the last couple of decades have seen improvements in the operative technique, anesthesia, perioperative care, and patient selection, resulting in a significant reduction in operative mortality rate to less than $4 \%$ for EPP in centers with significant experience. $^{26}$

Despite the theoretical advantage, radical surgery, however, failed to confer a survival advantage over debulking surgery in a multicentered Lung Cancer Study Group (LCSG) prospective trial. Between 1985 and 1988, the LCSG enrolled patients with biopsy proven MPM. Of the 83 patients accrued, only 20 were eligible for EPP. The remaining patients either underwent $\mathrm{P} /$ $\mathrm{D}$ or were treated nonsurgically. The postoperative mortality was $15 \%$ in the EPP group. While the recurrence-free survival was significantly longer for those who underwent EPP, there was no difference in overall survival among these three groups (level of evidence $2 b$ ). Furthermore, the authors did acknowledge that the observed difference in recurrence-free survival among the three groups might not be entirely attributable to the surgical approach because it was not adjusted for other prognostic indicators. ${ }^{27}$

\subsubsection{Multimodality Therapy}

Due to disappointing results with single-modality therapy, the consensus among experienced groups is that surgery is best combined with adjuvant chemotherapy, radiation, or a combination of these. Of all treatment approaches attempted, EPP, in combination with chemoradiation, has been most consistently associated with long-term disease-free survival and has provided for the greatest amount of cytoreduction.

\subsubsection{Evidence for Extrapleural Pneumonectomy and Adjuvant Therapy}

The largest experience to date supporting EPP followed by adjuvant therapy came from Brigham and Women's Hospital and Dana Farber Cancer Institute (BWH/DFCI). In this retrospective review of 183 patients, Sugarbaker and colleagues 
reported an overall median survival of 19 months, with 2-year and 5-year survivals of $38 \%$ and $15 \%$, respectively, with an impressive operative mortality of $3.8 \%$. A highly selected subset of 31 patients (roughly 15\% of the series) with epithelial type, negative surgical margins, and negative lymph nodes demonstrated an impressive median survival of 51 months, with 2- and 5-year survivals of $68 \%$ and $46 \%$, respectively. Survival correlated significantly with the stage of the disease. ${ }^{28}$

Similarly, in a phase II single-institution (MemorialSloan-Kettering Cancer Center; MSKCC) study of EPP (62 patients) versus P/D (5 patients) followed by hemithoracic radiation $(54 \mathrm{~Gy})$, the median survival in the EPP group was noted to be 17 months with an overall survival at 3 years of $27 \% .^{29}$ In contrast to the 51 months median survival in early stage in Sugarbaker's study, earlystage disease (stage I and II) "good" prognosis patients in this study had a median survival of 33.8 months only. A number of factors could account for the observed differences, namely trimodality therapy in Sugarbaker and colleagues study as opposed to bimodality therapy in Rusch and colleagues study; different staging criteria; etc. Due to the extremely small patient numbers, the survival was not assessed in the P/D group.

While both studies seem to support the aggressive surgical approach entailing EPP followed by some form of adjuvant therapy in early-stage MPM (level of evidence 2b), the efficacy of EPP over P/D has not been studied in a controlled clinical trial setting, making it difficult to draw any firm conclusions. In support of the above, Maziak and associates, in their systematic review of the surgical management of MPM, found only one prospective study by Pass and colleagues showing a significant difference in survival between the EPP (9.4 months) and P/D (14.5 months) treatment arms $(p=0.012)$. But patients were generally preselected for a specific surgical treatment in this series. ${ }^{30,31}$ None of the retrospective studies that included some form of adjuvant treatment reported a significant or consistent difference or advantage in median survival for EPP when compared to P/D. Unfortunately, interpretation of these trials is difficult, leaving the practitioner unsure as to which approach may offer the greatest benefit or least harm to the patient. Despite the relative lack of data and clarity some consensus and trends in patient management are emerging across centers of excellence for MPM treatment (discussed below).

\subsubsection{Evidence for Pleurectomy/Decortication with Adjuvant Therapy}

As most patients succumb to local tumor invasion rather than metastases, reducing the volume of tumor may be an important determinant of postoperative survival and can improve quality of life (QOL). Debulking surgery, whilst not curative, has the potential to obtain effective local control due to the protracted time before a significant amount of tumor re-accumulates to provoke symptoms. The choice of operation, EPP versus $P / D$, is dictated by several factors. The difference in the operative mortality between the two operations plays a major role in determining the suitability of one surgical approach over the other. The amount of disease and vital organs involvement further governs which operation is most suitable for a given patient. Patient factors such as inadequate cardiopulmonary reserve or comorbidities may preclude the more aggressive EPP approach. For this subgroup of patients, P/D offers a better therapeutic option. Yet, the real question as to whether P/D compared to nonoperative treatment prolongs survival has not been addressed in carefully controlled, prospective, randomized trials.

While retrospective studies have typically shown a survival advantage for patients undergoing surgery of any kind (P/D or EPP) versus nonoperated upon patients, these studies suffer from similar selection bias flaws in that the surgery arms of the studies all selected for better performance status low tumor burden patients, etc. As a debulking procedure, P/D inherently has a high local failure rate. Similar to the EPP experience, most institutions use combined modality therapy to reduce regional recurrences in $\mathrm{P} / \mathrm{D}$. The role of adjuvant chemotherapy, administered both locally and systemically, following P/D has also been investigated. The rationale for local intrapleural instillation of chemotherapy relies on the proclivity of MPM to remain localized even when advanced.

A significant amount of work has been performed by investigators at MSKCC in New York 
using intrapleural chemotherapy following $\mathrm{P} / \mathrm{D}$ in the treatment of MPM. In a phase II trial of P/ $\mathrm{D}$, Rusch and colleagues reported their experience with 27 patients with biopsy-proven MPM who underwent P/D followed immediately in the posteroperative period with intrapleural instillation of cisplatin and mitomycin via chest tubes and subsequently systemic administration of the two agents 3 to 5 weeks later. The median progression-free survival was 13.6 months with an overall survival at 2 years of $40 \%$ (with the median overall survival of 18.3 months). While the overall survival in this phase II trial was better than supportive care alone and comparable to other combined modality regimens, local relapse remained a major problem. Similar to the EPP experience, the investigators found that epithelial histology conferred significantly better survival than nonepithelial histology. ${ }^{32}$ One can cautiously conclude from this trial (feasibility nonrandomized trial) that in a select cohort with epithelial histology and an $\mathrm{R} 0$ resection with $\mathrm{P} / \mathrm{D}$, that $\mathrm{P} / \mathrm{D}$ offers a viable option in an adjuvant setting (level of evidence 2b). However, once again, trials such as these emphasize the need for multiinstituational and multidisciplinary approach to this rare disease entity in a phase III setting.

Memorial Sloan-Kettering Cancer Center investigators in their continual effort to better understand this disease to improve outcome reported their largest experience to date with $\mathrm{P} / \mathrm{D}$ followed by radiation. In this retrospective review, 123 patients between 1974 and 2003 underwent P/D. Postoperatively, the entire hemithorax was irradiated externally to a median dose of $42.5 \mathrm{~Gy}$ with a mixed photon electron beam technique to reduce damage to the underlying lung. Fifty-four patients also received intraoperative brachytherapy to any residual gross disease following P/D. In this series, the median survival and 2-year overall survival were 13.5 months and 23\%, respectively. Nonepithelial histology, left-sided disease, radiation dose less than $40 \mathrm{~Gy}$, and the use of implants were all noted to be negative predictors of overall survival in multivariate analysis. Despite this intensive treatment, $67 \%$ had local relapse. While this low-dose mixed photon-electron beam likely spared the underlying lung, it failed to produce long-term survivors or effective local control. The authors, therefore, concluded that adjuvant radiation is not an effective treatment option for eradicating residual disease following $\mathrm{P} / \mathrm{D}$ and that a more aggressive, that is, greater cytoreductive surgical approach was necessary ${ }^{33}$ (level of evidence $2 b$ ). Several other authors have reported a median survival ranging from 10 to 18 months with P/D followed by radiation again in small series with inherent biases.

We at University of California, San Francisco, have similarly performed a retrospective review of radical pleurectomy/decortication and intraoperative radiotherapy (IORT) followed by external beam radiation therapy with or without chemotherapy for diffuse malignant pleural mesothelioma. Of the 32 patients that were initially evaluated between 1995 and 2000, 26 that underwent successful radical P/D were entered into analysis. Twenty-four patients received IORT for a median dose of $15 \mathrm{~Gy}$. The same number of patients also received postoperative either threedimensional (3D) conformal RT (14 patients) or IMRT (10 patients). The median dose of external beam radiation was $41.4 \mathrm{~Gy}$. Some, but not all, patients were given platinum-based chemotherapy. A median overall survival of 18.1 months and an overall 2-year survival of $32 \%$ were demonstrated. Radical pleurectomy/decortication with aggressive radiotherapy with or without chemotherapy might offer an alternative treatment option to those who cannot tolerate extrapleural pneumonectomy. ${ }^{34}$ Yet, IORT is challenging to administer to sufficient tissue planes at risk (interlobar fissures, diaphragm, and pericardial/mediastinal pleura) and is not universally available. Whether the combined IORT radical P/D offered enhanced local control was not clear. Also, newer effective chemotherapy regimens with a pemetrexed backbone may achieve more for residual disease post P/D than IORT could offer. Finally, in these patients, although they benefited initially from improved pulmonary function, their pulmonary function gradually deteriorated with time (especially in the long-term survivors) such that the pneumonectomy-sparing value of radical P/D was diminished. In essence, they developed a slow-motion autopneumonectomy due to postoperative changes, injury or sacrifice of the phrenic nerve, and long-term radiation sequelae. Additionally, 
as shown in other series, higher dose external beam radiation especially when not $3 \mathrm{D}$ conformal and/or IMRT, has been associated with significantly higher toxicity.

The role of $\mathrm{P} / \mathrm{D}$ with intraoperative photodynamic therapy has also been investigated. Pass and colleagues in a phase III study randomized 63 patients to intraoperative PDT or not followed by postoperative immunochemotherapy. Fortyeight patients met the preoperative criteria for randomization. Twenty-three patients underwent $\mathrm{P} / \mathrm{D}$ and the remaining EPP. The median survival for this cohort was 14.4 months, compared with 7.7 months for those who were not debulked. Although pilot studies on the use of intraoperative photodynamic therapy as adjuvant therapy were promising, this phase III trial showed no apparent benefit for either progression-free (8.5 vs. 7.7 months) or overall survival (14.4 vs. 14.1 months) for intraoperative PDT. A trend, however, for longer survival was noted for those undergoing P/D as opposed to EPP (22 months vs. 11 months; $p=0.07$ ) regardless of intraoperative PDT (level of evidence 2b). ${ }^{35,36}$ Unfortunately, the study was not powered to detect the superiority of one surgical approach over the other.

\subsubsection{Evidence for Extrapleural Pneumonectomy and Neoadjuvant Therapy}

Neoadjuvant therapy is standard although somewhat controversial in certain locally advanced cancers such as stage IIIA (N2) non-small cell lung cancer. Recent advances in chemotherapy for MPM have led some groups to investigate the feasibility of induction chemotherapy prior to EPP in MPM patients as well. In a phase II trial, the MSKCC group enrolled patients with advanced disease to first undergo induction therapy with gemcitabine and cisplatin followed in 3 to 5 weeks by EPP and adjuvant $54 \mathrm{~Gy}$ radiation 4 to 6 weeks, postoperatively. The patients not only tolerated this regimen but their resectability rate was better (88\%) when compared with the historical resectability rate of $75 \%$ to $80 \%$. Despite the original concern that chemotherapy induced fibrosis might make the dissection more difficult, no operative mortality was noted. ${ }^{37}$

Similarly, a Swiss group reported no perioperative mortality and a median survival of 23 months in their series of 20 patients following induction chemotherapy with gemcitabine and cisplatin followed by EPP with or without adjuvant radiation. Interestingly, this was far superior to their own experience of EPP with adjuvant chemoradiation (a median survival of 13 months). ${ }^{38}$ A recently opened multicentered, prospective, randomized, phase III trial is underway in the United Kingdom comparing induction chemotherapy (pemetrexed-platinum doublet) alone to induction chemotherapy (same drugs) followed by EPP. While we await the results of this trial, the preliminary results appear promising and may translate into prolonged survival. Regardless, it does not appear that induction chemotherapy, especially regimens with pemetrexed, which is exceptionally well tolerated, increased surgical morbidity or mortality and is well tolerated even in the setting of EPP.

\subsection{The Debate}

In experienced centers, the surgical mortality associated with EPP is in the range of $3.8 \%$ to $5 \%$, comparable to $1.5 \%$ to $5 \%$ for $\mathrm{P} / \mathrm{D}$, which is a remarkable achievement given the frequent extent of disease encountered and the magnitude of the procedure being performed. Amazingly, in some centers the operative mortality with EPP is significantly less than the operative mortality for routine pneumonectomy for lung cancer $(5 \%-$ $8 \%)$. Therefore, operative mortality by itself should not preclude the more radical EPP approach. While EPP provides a more complete removal of gross disease, in patients with early disease both procedures allow for complete removal of gross disease. Extrapleural pneumonectomy may be more appropriate for those with advanced disease infiltrating the fissures and encasing the lung in whom $\mathrm{P} / \mathrm{D}$ may not get rid of all gross disease. Furthermore, EPP facilitates the feasibility of adjuvant radiotherapy. Pneumonectomy allows the entire hemithorax to be irradiated at a higher more effective dose (approx $50-60 \mathrm{~Gy}$ ) as opposed to $\mathrm{P} / \mathrm{D}$, where the underlying lung is at risk of radiation fibrosis, thus limiting the field and radiation dose. Extraplueral pneumonectomy, has better relapse-free survival. Unfortunately, despite these stated advantages, 
the evidence to date in support of EPP has failed to establish definitively a significant overall survival advantage over P/D. Of course, the real trial comparing the two approaches head to head prospectively has not been done. Yet, there is movement among MPM centers to consider such a trial. Now that there are multiple centers with teams of thoracic surgeons, medical and radiation oncologists who are experts in MPM, sufficient numbers of patients could be accrued both in the United States and Europe to answer the question.

Recent advances in chemotherapy in MPM have been encouraging. As mentioned previously, the combination of pemetrexed/cisplatin in unresectable disease has shown decent response. Currently, clinical trials are underway where this regimen is being studied in a neoadjuvant setting for those with resectable disease by EPP. There is a high likelihood that bulky disease may be reduced and render a patient a candidate for the lesser of the two aggressive procedures, that is, $\mathrm{P} / \mathrm{D}$, but still delivering a $\mathrm{R} 0$ resection. Our singleinstitution preliminary experience in a relatively small number of patients supports this hypothesis. We also have had anecdotal success in rendering those who were initially unresectable that after chemotherapy became amenable to resection.
In the final analysis, the optimal treatment of MPM remains to be determined by continued carefully planned and performed clinical trials. It is for this reason that patients with MPM should be referred to centers with established programs that are participating in multi-institutional efforts to allow the right questions to be asked and hopefully answered. For now, in the absence of irrefutable solid trial data, it is common practice for patients to be treated based on individual institutional biases and dependent on the multiple determinants, comorbidities, and performance status of the patient (Figure 54.1). The best evidence to date suggests that a subset of patients with favorable criteria such as epithelial histology, limited bulk of disease, and who are mediastinal (N2) node negative (i.e., early stage I or II) will benefit from enhanced local control and possible chance of cure with EPP and combined systemic chemotherapy and adjuvant radiation. The advent of molecular assessment of tumors and gene profiling should allow determination of the innate biological aggressiveness of an individual patient's tumor (perhaps regardless of other classic staging parameters such as $\mathrm{T}$ or $\mathrm{N}$ status) and this may help guide resection choice. Patients in whom long-term survival is less likely would benefit from non-pneumonectomy approaches that are still being developed. In the interim,

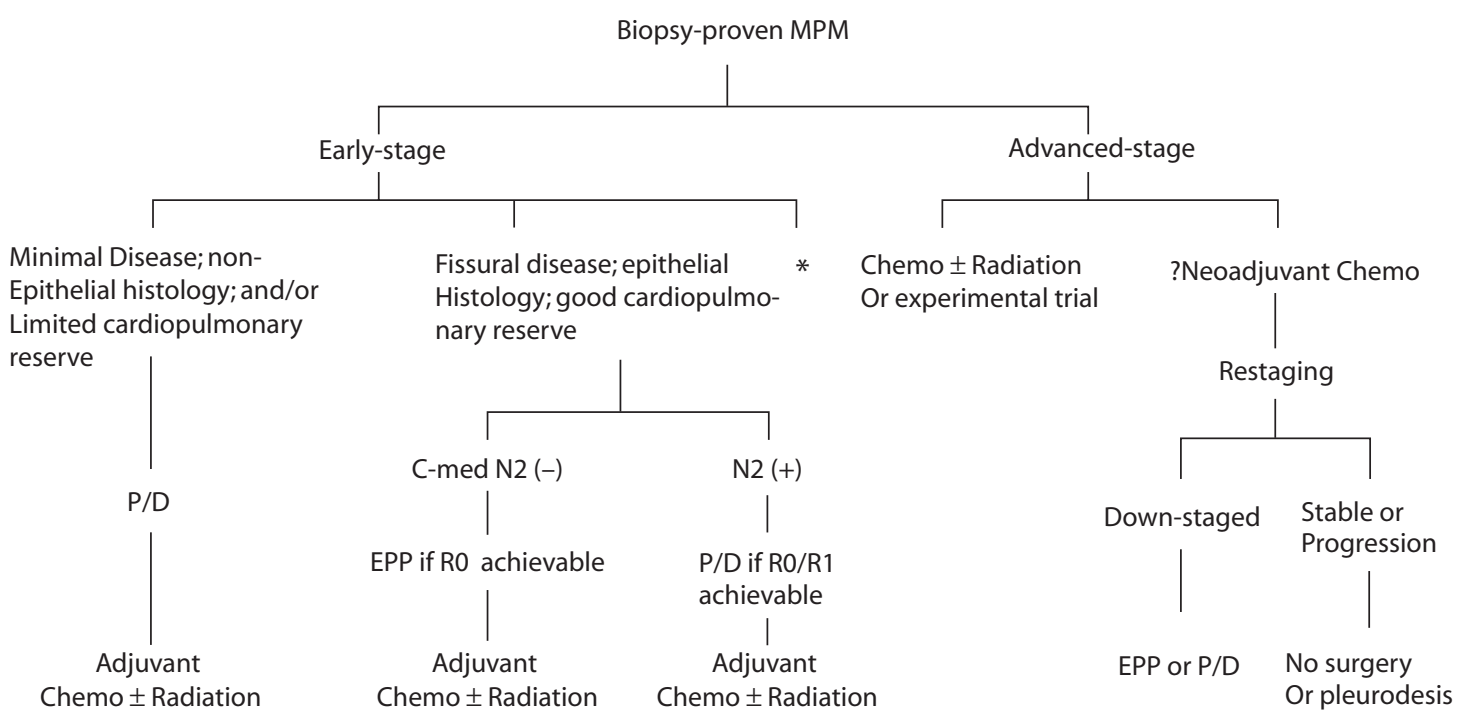

FIGURE 54.1. Algorithm for managing MPM. C-med, cervical mediastinoscopy; EPP, extrapleural pneumonectomy; N2, ipsilateral lymph node station; P/D, pleurectomy/decortication; ${ }^{*}$, neoadjuvant chemotherapy followed by EPP or P/D. 
current practice guidelines suggest that there is a role for radical P/D with intra-operative mediastinal node sampling as part of a combined modality approach in patients who cannot tolerate EPP and in whom an $\mathrm{R} 0$ or $\mathrm{R} 1$ resection can be delivered (Figure 54.1). Palliative ( $>\mathrm{R} 1) \mathrm{P} / \mathrm{D}$, on the other hand, probably has a limited role.

There is a role for radical P/D with intraoperative mediastinal node sampling as part of a combined modality approach in patients who cannot tolerate EPP and in whom an R0 or $\mathrm{R} 1$ resection can be delivered (level of evidence $2 \mathrm{~b}$; recommendation grade $\mathrm{B}$ ).

Palliative ( $>\mathrm{R} 1) \mathrm{P} / \mathrm{D}$ has a limited role in the management of MPM.

\section{References}

1. Enterline PE and VL Henderson. Geographic patterns for pleural mesothelioma deaths in the United States, 1968-81. J Natl Cancer Inst 1987;79:31-37.

2. Connelly RR, Spirtas R, Myers MH, et al. Demographic patterns for mesothelioma in the United States. J Natl Cancer Inst 1987;78:1053-1060.

3. Spirtas R, Beebe GW, Connelly RR, et al. Recent trends in mesothelioma incidence in the United States. Am J Ind Med 1986;9:397-407.

4. Devesa SS, Blot WJ, Stone BJ, et al. Recent cancer trends in the United States. J Natl Cancer Inst 1995;87:175-182.

5. Marom EM, Erasmus JJ, Pass HI, et al. The role of imaging in malignant pleural mesothelioma. Semin Oncol 2002;29:26-35.

6. Steele JPC. Prognostic factors in mesothelioma. Semin Oncol 2002;29:36-40.

7. Wang ZJ, Reddy GP, Gotway MB, et al. Malignant pleural mesothelioma: evaluation with CT, MR imaging, and PET. Radiographics 2004;24:105119.

8. Heelan RT, Rusch VW, Begg CB, et al. Staging of malignant pleural mesothelioma: comparison of CT and MR imaging. Am J Roentgenol 1999;172:1039-1047.

9. Patz EF, Shaffer K, Piwnica-Worms DR, et al. Malignant pleural mesothelioma: value of CT and MR imaging in predicting resectability. AJR Am J Roentgenol 1992;159:961-966.

10. Gerbaudo VH, Sugarbaker DJ, Britz-Cunningham $\mathrm{S}$, et al. Assessment of malignant pleural mesothe- lioma with (18)F-FDG dual-head gamma-camera coincidence imaging: comparison with histopathology. J Nucl Med 2002;43:1144-1149.

11. Benard F, Sterman D, Smith RJ, et al. Pro-gnostic value of FDG PET imaging in malignant pleural mesothelioma. J Nucl Med 2000;41:1443-1444.

12. Boutin C, Rey F. Thoracoscopy in the pleural malignant mesothelioma: a prospective study of 188 consecutive patients. Part I: diagnosis. Cancer 1993;72:389-393.

13. Baas P. Predictive and prognostic factors in malignant pleural mesothelioma. Curr Opin Oncol 2003;15:127-130.

14. Herndon J, Green M, Chahinian AP, et al. Factors predictive of survival among 337 patients with mesothelioma treated between 1984 and 1994 by the Cancer and Leukemia Group B. Chest 1998;113: 723-731.

15. Ruffie PA. Pleural mesothelioma. Curr Opin Oncol 1991;3:328-334.

16. DePangher-Manzini V, Brollo A, Franceschi S, et al. Prognostic factors of malignant mesothelioma of the pleura. Cancer 1993;72:410-417.

17. Byrne MJ, Davidson JA, Musk AW, et al. Cisplatin and gemcitabine treatment for malignant mesothelioma: a phase II study. J Clin Oncol 1999;17:2530 .

18. Taylor EC. Design and synthesis of inhibitors of folate-dependent enzymes as antitumor agents. Adv Exp Med Biol 1993;338:387-408.

19. Thodtmann R, Depenbrock H, Dumez H, et al. Clinical and pharmacokinetic phase I study of multitargeted antifolate (LY231514) in combination with cisplatin. J Clin Oncol 1999;17:3009-3016.

20. Calvert AH, Hughes AN, Calvert PM, et al. ALIMTA in combination with carboplatin demonstrates clinical activity against malignant mesothelioma in a phase I trial. Lung Cancer 2000;29: 73-74.

21. Vogelzang NJ, Rusthoven JJ, Symanowski J, et al. Phase III study of pemetrexed in combination with cisplatin versus cisplatin alone in patients with malignant pleural mesothelioma. J Clin Oncol 2003;21:2636-2644.

22. Baldini EH. External beam radiation therapy for the treatment of pleural mesothelioma. Thorac Surg Clin 2004;14:543-548.

23. Boutin C, Rey F, Viallat JR. Prevention of malignant seeding after invasive diagnostic procedures in patients with pleural mesothelioma: a randomized trial of local radiotherapy. Chest 1995;108: 754-758.

24. Butchart EG, Ashcroft T, Barnsley WC, et al. Pleuropneumonectomy in the management of diffuse 
diffuse malignant mesothelioma of the pleura. Experience with 29 patients. Thorax 1976;31:15-24.

25. Rusch VW. Pleurectomy/decortication in the setting of multimodality treatment of diffuse malignant pleural mesothelioma. Semin Thorac Cardiovasc Surg 1997;9:367-372.

26. Sugarbaker DJ, Jaklitsch MT, Bueno R, et al. Prevention, early detection, and management of complications after 328 consecutive extrapleural pneumonectomies. J Thorac Cardiovasc Surg 2004;128:138-146.

27. Rusch VW, Piantadosi S, Holmes EC, et al. The role extrapleural pneumonectomy in malignant pleural mesothelioma. A lung cancer study group trial. J Thorac Cardiovasc Surg 1991;102:1-9.

28. Sugarbaker DJ, Flores RM, Jaklitsch M, et al. Resection margins, extrapleural nodal status, and cell type determine postoperative long-term survival in trimodality therapy of malignant pleural mesothelioma: results in 183 patients. J Thorac Cardiovasc Surg 1999;117:54-65.

29. Rusch VW, Rosenzweig K, Venkatraman E, et al. A phase II trial of surgical resection and adjuvant high dose hemithoracic radiation for malignant pleural mesothelioma. J Thorac Cardiovasc Surg 2001;122:788-795.

30. Pass HI, Kranda K, Temeck BK, et al. Surgically debulked malignant pleural mesothelioma: results and prognostic factors. Ann Surg Oncol 1997;4: 215-222.

31. Maziak DE, Gagliardi A, Haynes AE, et al. Sugical management of malignant pleural mesothelioma: a systematic review and evidence summary. Lung Cancer 2005;48:157-169.
32. Rusch VW, Saltz L, Venkatraman E, et al. A phase II trial of pleurectomy/decortication followed by intrapleural and systemic chemotherapy for malignant pleural mesothelioma. J Clin Oncol 1994;12:1156-1163.

33. Gupta V, Mychalczak B, Krug L, et al. Hemithoracic radiation therapy after pleurectomy/decortication for malignant pleural mesothelioma. Int $J$ Radiat Oncol Biol Phys 2005;63:1045-1052.

34. Lee TT, Everett DL, Shu HG, et al. Radical pleurectomy/decortication and intraoperative radiotherapy followed by conformal radiation with or without chemotherapy for malignant pleural mesothelioma. J Thorac Cardiovasc Surg 2002;124: 1183-1189.

35. Friedberg JS, Mick R, Stevenson J, et al. A phase I study of Foscan-mediated photodynamic therapy and surgery in patients with mesothelioma. Ann Thorac Surg 2003;75:952-959.

36. Pass HI, Temeck BK, Kranda K, et al. Phase III randomized trial of surgery with or without intraoperative photodynamic therapy and postoperative immunochemotherapy for malignant pleural mesothelioma. Ann Surg Oncol 1997;4: 628-633.

37. Flores RM. Induction chemotherapy, extrapleural pneumonectomy, and radiotherapy in the treatment of malignant pleural mesothelioma: the Memorial Sloan-Kettering experience. Lung Cancer 2005;49:S71-S74.

38. Weder W, Kestenholz P, Taverna Cet al. Neoadjuvant chemotherapy followed by extrapleural pneumonectomy in malignant pleural mesothelioma. J Clin Oncol 2004;22:3451-3457. 